\title{
Overexpression of human CUTA isoform2 enhances the cytotoxicity of copper to HeLa cells
}

\author{
Jingchun Yang ${ }^{1}$, Qiang $\mathrm{Li}^{1}$, Huirong Yang ${ }^{1}$, Lichong Yan², Liu Yang ${ }^{1}$ and Long $\mathrm{Yu}^{1} \bowtie$ \\ ${ }^{1}$ State Key Laboratory of Genetic Engineering, School of Life Science, Fudan University, Shanghai, P. R. China; \\ ${ }^{2}$ School of Life Science, Soochow University, Soochow, P. R. China
}

Received: 31 January, 2008; revised: 13 May, 2008; accepted: 11 June, 2008

available on-line: 14 June, 2008

\begin{abstract}
Copper, an essential transient element, can be toxic to cells when present in excess. Altered copper homeostasis is involved in pathological events of many diseases. Human CUTA isoform2 is a member of cation tolerance protein (CutA1) family. In this study, we examined the effect of CUTA isoform2 overexpression on copper toxicity. It was shown that overexpressed CUTA isoform2 sensitized HeLa cells to copper toxicity by promoting copper-induced apoptosis. The inhibition effect of excessive copper on cell proliferation was also enhanced by overexpressed CUTA isoform2. So CUTA isoform2 was implicated to be involved in the cytotoxicity of copper.
\end{abstract}

Keywords: copper toxicity, CUTA isoform2, overexpression, copper-induced apoptosis, cell proliferation

\section{INTRODUCTION}

Excessive copper can damage lipids and proteins (Gunther et al., 1995; White et al., 1999; Urbański \& Beręsewicz, 2000) and induce oxidative stress in cells. The toxicity induced by copper imbalance is thought to be involved in the pathological events of many neurodegenerative diseases such as Alzheimer's disease, Parkinson's disease, amyotrophic lateral sclerosis and prion disease (Butterfield \& Boyd-Kimball, 2004; Poon et al., 2004). Proteins such as amyloid precursor protein (APP) and prion protein, associated with these diseases displayed copper-binding capability. The cytotoxicity of copper has been used in cancer chemotherapy. Copper-based drugs called casiopeinas exhibit higher antineoplastic potency than cisplatin in in vitro and in vivo studies of several tumor cell lines (De Vizcaya-Ruiz et al., 2000). Thus the investigation on molecular mechanisms of copper toxicity will be important for understanding its role in disease progression and in cancer therapy.
The human CUTA gene has five variant transcripts encoding three isoforms with different aminoterminal sequences. CUTA isoform2 (gi: 62526022) with the shortest amino-terminal sequence has been implicated in anchoring acetylcholinesterase to the cell surface (Navaratnam et al., 2000; Perrier et al., 2000). However, the CUTA isoform2 has a conserved CutA1 domain, which is characteristic for the CutA1 family proteins. CutA1 protein is widespread in bacteria, plants, and animals, including humans, and was originally identified in a gene locus of Escherichia coli called cutA involved in copper tolerance. Molecular genetic studies on the E. coli cutA locus showed that some mutations lead to copper sensitivity due to its increased uptake (Fong et al., 1995). However, the special function of CutA1 in E. coli is still unknown. Recently, it was reported that many CutA1 proteins had copper binding capacity (Arnesano et al., 2003; Burkhead et al., 2003; Tanaka et al., 2004). Intriguingly, copper could induce reversible aggregation of CutA1 protein in Pyrococcus horikoshii (Tanaka et al., 2004). CUTA isoform2, human CutA1

$\triangle$ Corresponding author: Long Yu, State Key Laboratory of Genetic Engineering, School of Life Science, Fudan University, Handan road 220, Shanghai 200433, P. R. China; phone: 86-21-6564 3954; fax: 86-21-6564 3404; e-mail: Longyu@fudan.edu.cn Abbreviations: AChE, acetylcholinesterase; APP, amyloid precursor protein; $\mathrm{B} 2 \mathrm{M}, \beta_{2}$-microglobulin; CutA, cation tolerance protein; MTT, 3-(4,5-dimethylthiazole-2-yl)-2,5diphenyl tetrazolium bromide; PBS, phosphate-buffered saline; RT$\mathrm{PCR}$, reverse transcription-polymerase chain reaction; 7-AAD, 7-amino-actinomycin. 
protein, has the conserved CutA1 domain, so there seemed to be a link between CUTA isoform2 and copper homeostasis. In this work, we examined the effect of CUTA isoform2 overexpression on copper toxicity.

\section{MATERIALS AND METHODS}

Cell culture. CUTA isoform2 stable transformed HeLa cells and control cells were constructed previously (Yang et al., 2007). Briefly, the coding sequence of CUTA isoform2 gene (AF106943) was cloned and inserted into pcDNA3.1 vector. The stably transformed HeLa cells were selected by G418 resistance. The HeLa cells stably transfected with CUTA isoform2 were named HeLa-CUTA, while the control cells were named HeLa-vector.

Semiquantitative reverse transcription-PCR. The total nuclear RNA was isolated from the HeLa cells by Trizol, and reverse transcribed into the first strand cDNA using SuperScript ${ }^{\mathrm{TM}}$ III first-strand synthesis system kit (Invitrogen, USA). Equal amounts of cDNA template were used in the subsequent PCR with primer 1 (5'-taagaattcatgccggcgctgctgcctg- $\left.3^{\prime}\right)$ and primer 2 (5'-gcaaagctttggcaggactgtgatagagtc- $\left.3^{\prime}\right)$ for CUTA gene, primer 3 (5'-atgagtatgcetgccgtgtgaac- $\left.3^{\prime}\right)$ and primer 4 (5'-tgtggagcaacctgctcagatac- $\left.3^{\prime}\right)$ for $\beta_{2}$-microglobulin (B2M) gene. Twenty microlitres of each PCR product was separated on a $2 \%$ agarose gel and detected by ultraviolet light.

Cell survival by MTT assay. The MTT colorimetric assay (Promega, USA) was used to assess the sensitivity of the cells to copper toxicity. Exponentially growing cells were trypsinized and harvested. Then cells of equal numbers in $100 \mu \mathrm{l}$ of medium were inoculated into each well of a 96-well microplate. After incubating overnight, the solution containing $\mathrm{CuSO}_{4}$ was added to the cultures, and they were incubated for the indicated time. Surviving cells from each well were treated with $120 \mu \mathrm{l} /$ well MTT reagents two hours prior to harvest. Absorbance at $570 \mathrm{~nm}$ was recorded using an enzymelinked immunosorbent assay plate reader.

Cell proliferation assay. The stably transformed HeLa cell lines were seeded in 96-well plates at a density of 600 cells per well. After $24 \mathrm{~h}$ of incubation the medium was replaced with the normal medium or medium containing $\mathrm{CuSO}_{4}$. Cell proliferation was determined from day 1 to day 5 by MTT cell viability assay.

Apoptosis analysis. The stably transformed HeLa cells were dispensed into 6-well plates $\left(4 \times 10^{4}\right.$ cells per well) and treated with appropriate concentration of $\mathrm{CuSO}_{4}$. After indicated time of $\mathrm{CuSO}_{4}$ treatment, cells were harvested, washed twice with PBS ( $\mathrm{pH}$ 7.4), resuspended in $1 \times$ staining buffer, and stained with Annexin V-PE and 7-AAD for $15 \mathrm{~min}$ according to the manufacturer's protocol (BD Biosciences ${ }^{\circledR}$, USA). Fluorescence was measured with bandpass $620 / 22$ and $575 / 24 \mathrm{~nm}$ filters by BD FACSCanto $^{\mathrm{TM}}$ flow cytometer and the extent of apoptosis was determined.

Statistical analysis. Data were statistically tested using the $t$ test for independent samples (Excel; Microsoft, Redmond, WA, USA).

\section{RESULTS AND DISCUSSION}

Copper is highly toxic when it exceeds cellular needs and accumulates in the cell. In order to examine the role that human CUTA isoform2 plays in copper toxicity, we used two stable cell lines HeLa-vector and HeLa-CUTA, which had been constructed and maintained previously (Yang et al., 2007). The overexpression of CUTA in stable cells was confirmed by RT-PCR. The cDNA was obtained and used as a template for PCR amplification. The primer used in PCR amplification targeted the sequence located in the open reading frame of CUTA isoforms. As shown in Fig. 1, although a specific fragment corresponding to CUTA cDNA could be detected in both HeLa cell lines, HeLa-CUTA cells showed increased accumulation of CUTA-specific mRNA compared to the control cells because of the foreign CUTA gene expression. As a native control, equal quantity of cDNA corresponding to the $\mathrm{B} 2 \mathrm{M}$ gene was detected in two cell lines. CUTA isoform2 was found to be overexpressed in HeLa-CUTA cells. The overexpressed CUTA isoform 2 protein in HeLaCUTA was tested by anti-Myc antibody previously (Yang et al., 2007).

To analyze the effect of CUTA isoform2 overexpression on cytotoxicity induced by excessive copper, the difference in susceptivity to copper toxicity between the two stable cell lines was compared by examining the dose-response of the

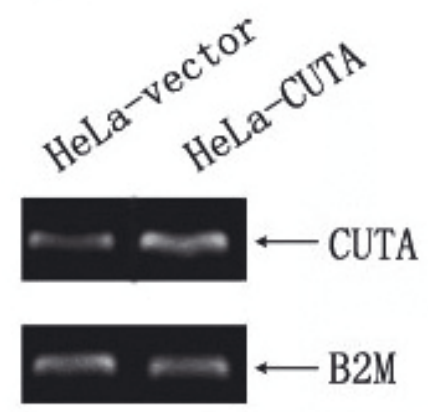

Figure 1. Overexpression of CUTA isoform2 in stable cells assessed by RT-PCR.

RNA extracted from HeLa-CUTA or HeLa-vector cells was used for RT-PCR. The specific primers were used to amplify the cDNA of CUTA or $\beta_{2}$-microglobulin (B2M) gene. 


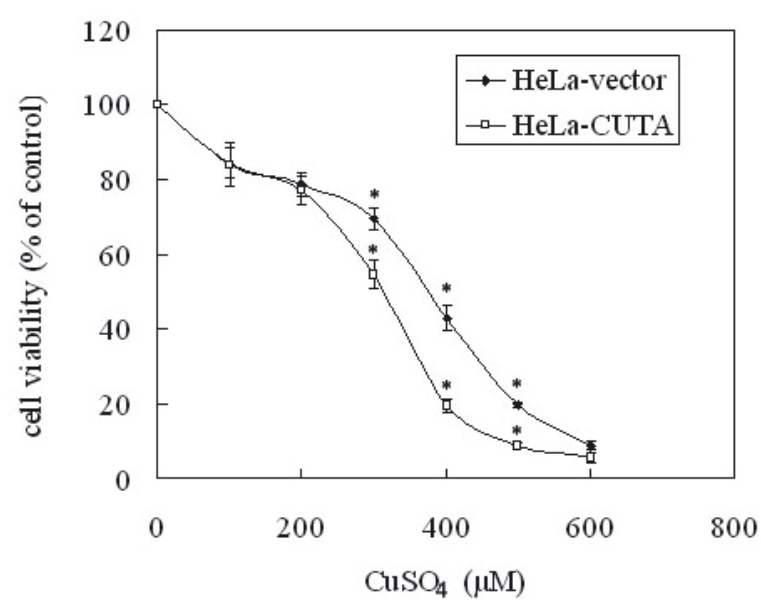

Figure 2. The cell viability decreased by excessive copper.

HeLa-vector and HeLa-CUTA cells were grown in complete medium or medium to which $\mathrm{CuSO}_{4}$ was added. Cell viabilities were determined by MTT assay following 3-day incubation in the presence of $\mathrm{Cu}^{2+}$. Each sample was prepared in six repeat wells. Percent survival was determined from the number of cells grown in copper-added medium as compared to the same cell line grown in medium without $\mathrm{Cu}^{2+}$, expressed as $100 \%$. *Significant difference in cell viability $(P<0.01)$

two cell lines to copper (Fig. 2). In this dose-response assay, both HeLa-CUTA cells and the control HeLa-vector cells showed decreased cell viability with enhanced copper concentration. However, the percentage of decrease in cell viability in HeLa-CUTA cells was significantly higher than that of HeLa-vector cells. So HeLa-CUTA cells had a significantly lower percentage of cell viability than that of the control cells under $\mathrm{CuSO}_{4}$ concentrations $(300,400,500 \mu \mathrm{M})\left({ }^{*} P<0.01\right)$. This suggested that overexpression of CUTA isoform2 sensitized the HeLa-CUTA cells to copper toxicity. Overexpression of CUTA isoform2 also sensitized the cell to the toxicity from manganese and zinc (not shown). It seems that the function of CUTA isoform2 is universal to the divalent cation. To further characterize cell death when the cells were exposed to copper, the differences in apoptosis between HeLa-CUTA and HeLa-vector cells were examined. Quantification of dose and time dependency was done by monitoring the percentage of apoptotic cells (Fig. 3A, B). A remarkable increase of apoptosis cell percentage was observed in HeLa-CUTA cells with the increased copper concentration and prolonged time of treatment. Comparatively, the increase of apoptosis cells percentage is not so evident in the control HeLa-vector cells. These data showed that exposure to excessive copper resulted in more apoptotic cell death in HeLa-CUTA cells than in the controls. CUTA isoform2 overexpression promoted the copper-
A

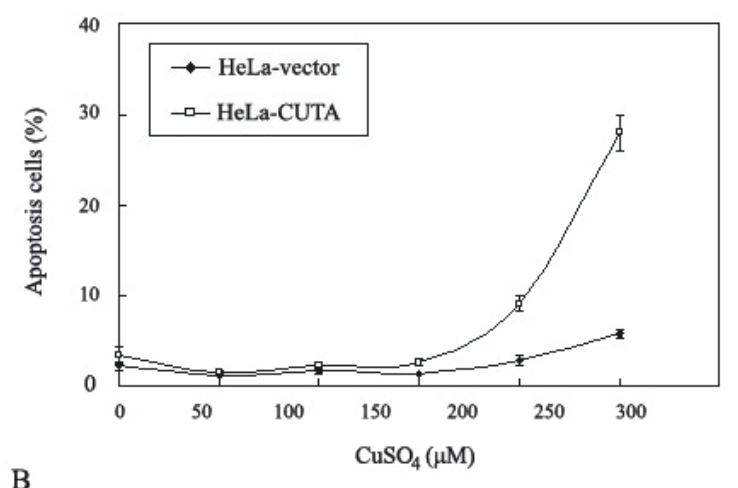

B

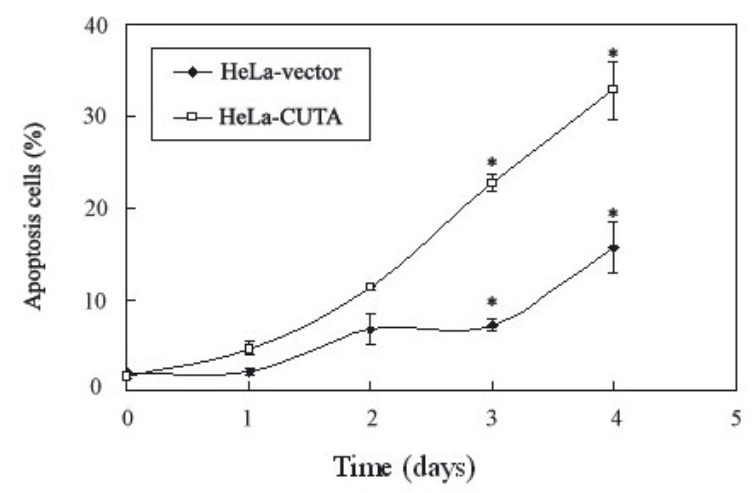

Figure 3. Copper-induced apoptosis detected by Annexin V-PE/7-AAD staining.

The stably transformed HeLa cells were treated with $\mathrm{CuSO}_{4}$. Then Annexin V-PE/7-AAD staining followed by flow cytometry was performed to determine levels of apoptosis in response to copper treatment. (A) Doseresponse curve for induction of apoptosis by copper in HeLa cells. Cells were incubated for 4 days in culture media plus various amounts of $\mathrm{CuSO}_{4}$ before apoptosis assay by flow cytometry. (B) Time-response curve for induction of apoptosis by copper in HeLa cells. Stably transformed HeLa cells were incubated with $250 \mu \mathrm{M} \mathrm{CuSO}_{4}$, and then the apoptosis assay of the cells was performed on the indicated time. At each time or concentration point three repeat wells were measured. *Significant difference in cell apoptosis $(P<0.05)$.

induced apoptosis and then sensitized the HeLa cells to copper toxicity.

Excessive copper could not only decrease the cell viability but also inhibit the rate of cell proliferation. To compare the effect of excessive copper on cellular growth rates, the cell growth curve of HeLa-CUTA and HeL-vector cells was assessed by MTT assay in the absence or presence of copper. As shown in Fig. 4, medium containing a low concentration of copper hardly affected the cell growth compared to the normal medium. Under this culture condition, HeLa-CUTA cells showed a similar growth rate to that of HeLa-vector cells. However, treatment with 200 and $250 \mu \mathrm{M} \mathrm{CuSO}{ }_{4}$ obviously suppressed cell growth in both cell lines. At these copper concentrations, we could see the prolifera- 

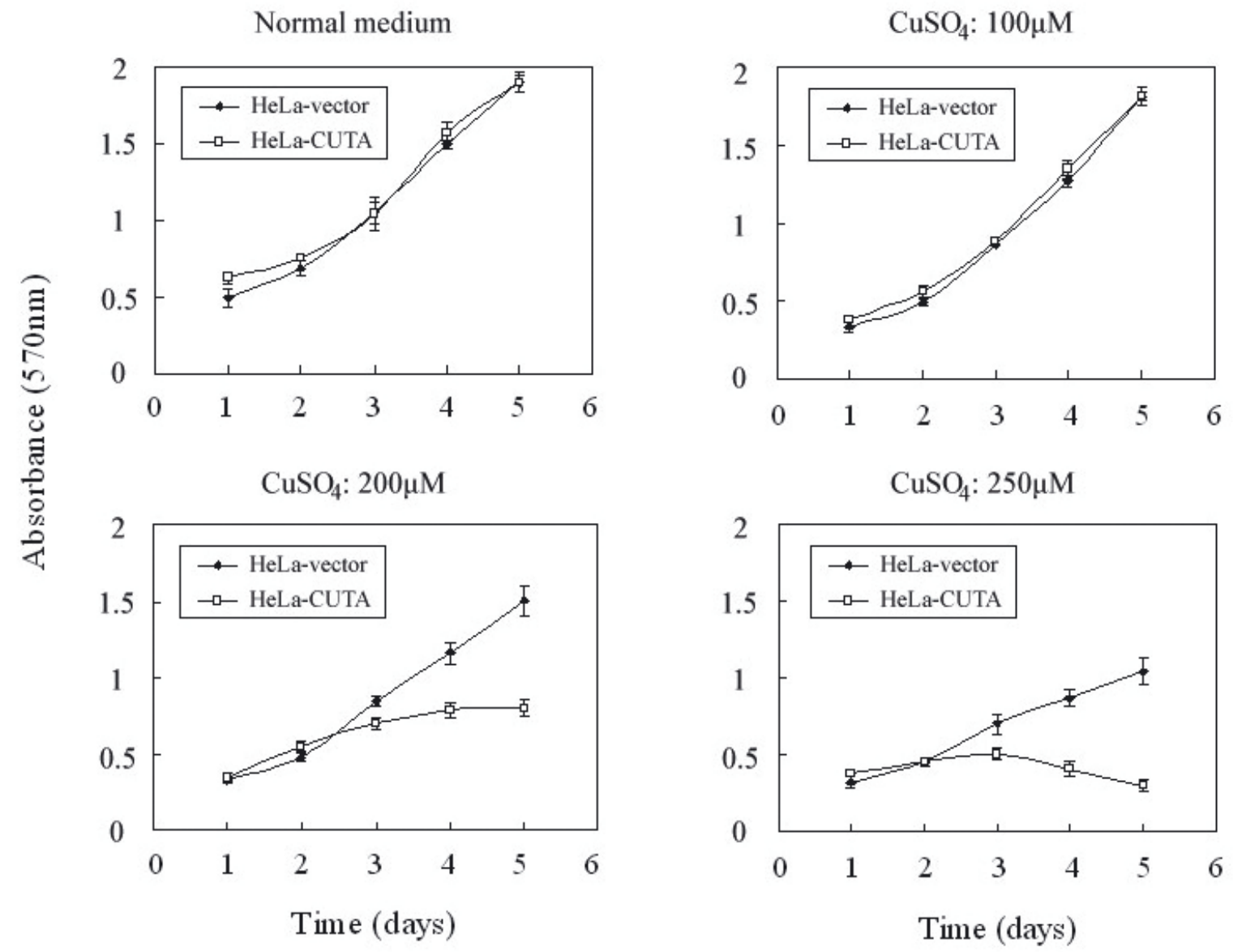

Figure 4. Cell proliferation measured by MTT assay.

The two cell lines of HeLa-CUTA and HeLa-vector were cultured with normal medium or medium containing different concentrations of $\mathrm{CuSO}_{4}(100,200,250 \mu \mathrm{M})$. Cell proliferation was monitored by MTT cell viability assay from day 1 to day 5. Each data shown in the figure was expressed as the mean value with the standard error of six repeat wells.

tion rate of HeLa-CUTA cells was significantly lower than that of the controls by comparing the slope of the growth curve in the log phase. Furthermore, the growth curve slope of HeLa-CUTA cells became negative when the cells had been incubated with 250 $\mu \mathrm{M} \mathrm{CuSO}_{4}$ for 3 days. This indicated that CUTA isoform2 overexpression in HeLa-CUTA cells enhanced copper-induced inhibition of cell growth.

Overexpression of CUTA isoform2 not only sensitized HeLa cells to copper toxicity but also enhanced copper-induced inhibition of cell proliferation. So CUTA isoform2 was implicated as playing important role in copper toxicity. The study will be useful for further analysis of the mechanism underlying copper toxicity. Furthermore, AChE, which had been implicated to be anchored in neuron membrane through CUTA isoform2, is a component of senile plaques of Alzheimer's disease and forms a stable complex with other components through its peripheral anionic site (Small et al., 1996; Soreq \& Seidman, 2001; Greenfield \& Vaux, 2002). Thus it seems that there is an intriguing link between
CUTA isoform2 and Alzheimer's disease. Our study will also be helpful for the investigation on the role copper toxicity plays in Alzheimer's disease. In addition, the expression of CUTA isoform2 in cancer will be an important instrument for cancer chemotherapy based on copper toxicity since overexpression of CUTA isoform2 could sensitize the cells to copper toxicity.

\section{Acknowledgements}

This work was supported by the National 973 program of China, 863 projects of China and the National Natural Science Foundation of China (30024001).

\section{REFERENCES}

Arnesano F, Banci L, Benvenuti M, Bertini I, Calderone V, Mangani S, Viezzoli MS (2003) J Biol Chem 278: 459996006 . 
Burkhead JL, Abdel-Ghany SE, Morrill JM, Pilon-Smits EA, Pilon M (2003) Plant J 34: 856-867.

Butterfield DA, Boyd-Kimball D (2004) Brain Pathol 14: 426-432.

De Vizcaya-Ruiz A, Rivero-Muller A, Ruiz-Ramirez L, Kass GE, Kelland LR, Orr RM, Dobrota M (2000) Toxicol In Vitro 14: 1-5.

Fong ST, Camakaris J, Lee BT (1995) Mol Microbiol 15: 1127-1137.

Greenfield S, Vaux DJ (2002) Neuroscience 113: 485-492.

Gunther MR, Hanna PM, Mason RP, Cohen MS (1995) Arch Biochem Biophys 316: 515-522.

Navaratnam DS, Fernando FS, Priddle JD, Giles K, Clegg SM, Pappin DJ, Craig I, Smith AD (2000) J Neurochem 74: 2146-2153.

Perrier AL, Cousin X, Boschetti N, Haas R, Chatel JM, Bon S, Roberts WL, Pickett SR, Massoulie J, Rosenberry TL, Krejci E (2000) J Biol Chem 275: 34260-34265.
Poon HF, Calabrese V, Scapagnini G, Butterfield DA (2004) Clin Geriatr Med 20: 329-359.

Small DH, Michaelson S, Sberna G (1996) Neurochem Int 28: 453-483.

Soreq H, Seidman S (2001) Nat Rev Neurosci 2: 294-302.

Tanaka Y, Tsumoto K, Nakanishi T, Yasutake Y, Sakai N, Yao M, Tanaka I, Kumagai I (2004) FEBS Lett 556: 167-174.

Urbański NK, Beręsewicz A (2000) Acta Biochim Polon 47: 951-962.

White AR, Multhaup G, Maher F, Bellingham S, Camakaris J, Zheng $\mathrm{H}$, Bush AI, Beyreuther K, Masters CL, Cappai R (1999) J Neurosci 19: 9170-9179.

Yang J, Yang H, Yan L, Yang L, Yu L (2007) Mol Biol Rep (in press). 